\title{
Barries in precision medicine implementation among Advanced Nonsquamous Cell Lung Cancer- patients: A Real-World-Evidence Scenario
}

Flávia A. Duarte ( $D$ flavia.amaral@outlook.com )

Oncoclínicas do Brasil

Carlos Gil Ferreira

Oncoclínicas Institute

Rodrigo Dienstmann

Oncoclínicas Group

Bruno L. Ferrari

Oncoclínicas do Brasil

Matheus Costa e Silva

Oncoclínicas Group

Pedro Narazeth A. Junior

Oncoclínicas Group

Paulo Guilherme 0. Salle

Roberto Alvarenga Institute

Paulo Henrique C. Diniz

Oncoclínicas do Brasil

\section{Research Article}

Keywords: Precision medicine, drug access, non-small cell lung cancer

Posted Date: September 27th, 2021

DOl: https://doi.org/10.21203/rs.3.rs-927396/v1

License: (1) This work is licensed under a Creative Commons Attribution 4.0 International License.

Read Full License 


\section{Abstract \\ BACKGROUND}

Precision oncology has a prominent role in nonsquamous non-small cell lung cancer (nsNSCLC) treatment progress. This study aimed to investigate not-cost-related factors involved in the use of the molecular profile in clinical practice and the influence of its availability on clinical decisions.

\section{METHODS}

nsNSCLC patients who underwent molecular testing in a private referral Brazilian center were identified. The primary endpoint was the interval from the diagnosis of advanced nsNSCLC to the characterization of the molecular profile. Other outcomes, focusing on the biomarker tissue journey, were also assessed, as well as the choice of therapy and changes in the treatment upon the testing results.

\section{RESULTS}

In this cohort $(n=78)$, the median time between the advanced nsNSCLC diagnosis and biomarker characterization was 40.5 days (range, 29.5-68.5). The median interval between the diagnosis and the test request was longer than the interval between the request and the results (respectively 29.0 versus 12.0 days; $p<0.001)$. At the treatment initiation, $51 \%(36 / 71)$ of the patients did not have their full driver mutations panel results available. Of these, $42 \%(15 / 36)$ had a targetable alteration identified later on. Among patients harboring a targetable alteration, only $46 \%(n=13 / 28)$ received a tyrosine kinase inhibitor (TKI) as first-line therapy. The median time to the TKI initiation was even longer than the median time to all treatment initiation (92.0 versus 40.0 days).

\section{CONCLUSIONS}

Our data show a long median time from advanced nsNSCLC diagnosis and the availability of the biomarker testing in medical practice, which impacted the choice of a non-personalized therapy as the first-line and the necessity of treatment changes after the final report.

\section{Background}

Lung cancer ranks as the leading cause of cancer worldwide, and accounts for the largest number of cancer deaths (1.8 million deaths, $18.4 \%$ of the total). In 2018, approximately 2.1 million diagnoses were estimated, representing $11.6 \%$ of the total cancer incidence burden. Of those, $3.27 \%$ were observed in South American ${ }^{1}$. Non-small cell lung cancer (NSCLC), which comprises $80-85 \%$ of lung malignancies, is the most frequent histology. The knowledge of the tumor molecular pathways as well as the interaction between tumor cells and the immune system led to the development of innovative therapies such as 
targeted agents and immune checkpoint inhibitors. These therapeutical advances improved the outcomes even for patients diagnosed at advanced stage ${ }^{2}$. Certainly precision oncology has a prominent role in the remarkable progress in this scenario.

Since targeted therapy use is tailored by molecular findings, such as specific genetic alterations (e.g. EFGR, ALK, ROS-1) and PD-L1 expression, biomarker testing becomes mandatory to guide the therapeutic decisions on the lung cancer approach. In a real-world scenario, there are still many challenges and barriers to be overcome in order to derive the most benefit for the patients. The high cost of such innovative treatments have traditionally been recognized as a major issue. However, the access to the molecular tests is certainly another important matter.

In Brazil, where lung cancer is also among the most common malignancies ${ }^{3}$, the pharmaceutical industry-sponsored programs have been a useful tool to overcome barriers in the molecular testing access, as in other low and middle-income countries. Regardless of this support, it is well known that the access to molecular testing is limited and data on the frequency of driver mutations is still scarce 4 .

Apart from these cost-related factors, issues concerning molecular testing itself, such as insufficient tumor samples, inadequate tumor tissue preservation and logistics delays may impact the prompt identification of a biomarker, which is essential for personalized therapy and may impact the clinical outcomes. The concerns grow since there is evidence suggesting the choice of appropriate targeted treatment in the first-line setting as a determinant of improved clinical outcomes, including best response, quality of life, favorable toxicity profile, and progression-free survival ${ }^{5-7}$.

Thus, this study aimed to investigate in a Real World Evidence-scenario (RWE) the use of the NSCLC molecular profile in clinical practice, the impact of the availability of these testings in clinical decisions and to identify not-cost related barriers to the applicability of the best evidence-based targeted treatment in a Brazilian population.

\section{Methods}

\section{Study design and cohort}

This is a non-interventional, single-center, retrospective study. We included patients with histologically confirmed locally advanced or metastatic non-squamous NSCLC (nSNSCLC), who underwent molecular testing, between November 2015 and February 2020 in a private referral Brazilian center.

Demographic and clinical data were retrospectively collected from medical records. The molecular profile consists of testing alterations such as EGFR, ALK, ROS1, BRAF, and KRAS. For inclusion in this cohort, it was not necessary to perform the tests for all of these genes. The PD-L1 expression was also registered, when available. 
Exclusion criteria were: age < 18 years, mixed histology (i.e. adenosquamous carcinoma). We also excluded patients with recurrent disease whose biopsy at recurrence was not available, and patients whose date of the biomarker panel conclusion was missing.

The study was approved by an independent Ethics Committee (4.171.310), and the protocols were in accordance with the ethical guidelines of the 1975 Helsinki Declaration. Due to the retrospective nature of this study, the local Human Subjects Committee approved the waiver of participants' free and informed consent.

\section{Endpoints}

The primary endpoint was the interval from the diagnosis of advanced nsNSCLC to the characterization of the molecular profile. It comprehends the period between the date of histologic diagnosis or disease recurrence and result of the last biomarker test performed.

The secondary endpoints were the time between the diagnosis or disease recurrence and the testing request, the time between the testing request and the final report, the proportion of patients with confirmed driver mutations before the first-line treatment decision, and the proportion of patients whose treatment changed due to the testing results.

To evaluate the suitability of treatment decisions, the data of drug approvals by the Brazilian national health surveillance agency (ANVISA) were also taken into consideration.

Given the great variability of techniques available for molecular testing, harbouring diffent perfomances and time to results avalilability, the assays used to evaluate the presence of driver mutations, fusions and translocations were also investigated. Similary, the different immunohistochemistry technics used to analyze PD-L1 expression were also recorded (table 3).

\section{Statistical analyses}

Descriptive statistics were used to summarize the data. A normality test (Shapiro-Wilk) was performed for each continuous variable. Categorical data were presented as frequency and percentages, and continuous data expressed as medians and interquartile ranges. If normality assumed continuous variables were expressed as means and standard deviations. As the study was descriptive, estimation of sample size or statistical power was not applicable.

For comparisons between dependent samples, the Wilcoxon Signed Ranks was used. Statistical significance was assumed at $p<0.05$. Statistical analyses were carried out using SPSS ${ }^{\circledR}$ software, version 20 (SPSS, Chicago, IL).

\section{Results}

Patients characteristics 
In this cohort, 78 eligible patients were identified. Their demographic, clinical, and histopathological characteristics are summarized in Table 1. The mean age at initial diagnosis of was 69 years (Range 40 to 92 ). Approximately half of patients were male (49.0\%) and former or current smokers (49\%).

Table 1: Demographic characteristics

\begin{tabular}{|lll|}
\hline Variable & Mean/Frequency \\
\hline Age at diagnosis & 69 years (range 40-92) \\
\hline ECOG & 0 & $32 \%$ \\
$(n=76)$ & 1 & $59 \%$ \\
& 2 & $9 \%$ \\
\hline $\begin{array}{lll}\text { Smoking } \\
(n=74)\end{array}$ & Never & $51 \%$ \\
& Current & $12 \%$ \\
\hline Gender & Former & $37 \%$ \\
$(n=78)$ & Memale & $51 \%$ \\
\hline
\end{tabular}

Abbreviation: ECOG, Eastern Cooperative Oncology Group Scale of Performance Status ${ }^{€}$ value in median (interquartile range).

Regarding EGFR mutations, it was identified in 26 (33\%) patients. Among them, 92.9\% ( $n=24 / 26)$, harbored a common sensitizing mutation. Considering the patients whose tumor were tested for ALK translocation $(n=58 ; 74 \%)$, this molecular alteration were presented only in three $(5 \%)$. Likewise, ROS1 fusions were identified in only one patient (2.8\%). KRAS and BRAF mutations were evaluated in 47 (60.3\%) patients and were detected in 11 and five patients, respectively (Figure 1A and 1B).

Considering the patients in which PDL-1 expression was analyzed $(n=55), 30$ patients (55\%) had no PDL1 expression and only 9 (16\%) had a strong expression (>50\%) (Figure 1C). Of these high expressors, two harbored a concomitant BRAF punctual mutation, while other one presented a ROS1 fusion.

\section{Biomarker testing}

The majority of the patients (61\%) were not tested for the all six biomarker investigated (Figure 1D). There were a great variability of assays used especially for EGFR mutations. Next Generation Sequencing (NGS) was used only in $4 \%$ (3 cases). For ALK translocations assessment, almost all cases (93\%) used an immunohistochemistry assay. For PD-L1 expression testing, there were three available antibodies and the most frequent was the Ventana SP263 (63\% of all cases). The assays for biomarker testing were summarized in the Table 2. 
Table 2: Biomolecular assays used in the molecular testing

\begin{tabular}{|lll|}
\hline Biomarker & Type of Test & Frequency \\
\hline EGFR & rtPCR & $39 \%$ \\
\cline { 2 - 3 }$(n=77)$ & NGS hotspot & $56 \%$ \\
\cline { 2 - 3 } & Liquid Biopsy & $1 \%$ \\
\cline { 2 - 3 } ALK & NGS & $4 \%$ \\
$(n=58)$ & IHC & $93 \%$ \\
\cline { 2 - 3 } & FISH & $5 \%$ \\
\hline PD-L1 & NGS & $2 \%$ \\
\cline { 2 - 2 }$(n=56)$ & SP263 & $29 \%$ \\
\cline { 2 - 3 } & E1L3N & $62 \%$ \\
\hline ROS1 & FISH & $97 \%$ \\
\cline { 2 - 3 }$(n=36)$ & NGS & $3 \%$ \\
\hline KRAS & NGS hotspot & $96 \%$ \\
\cline { 2 - 3 }$(n=47)$ & NGS & $4 \%$ \\
\hline BRAF & NGS hotspot & $96 \%$ \\
$(n=47)$ & NGS & $4 \%$ \\
\cline { 2 - 3 } & & \\
\hline
\end{tabular}

Abbreviation: rtPCR, real time polymerase chain reaction; NGS, next generation sequencing; IHC, immunohistochemistry; FISH, fluorescence in situ hybridization.

Time for testing results and first line treatment decisions

The median time between the advanced nsNSCLC diagnosis and the final biomarker characterization was 40.5 days (range, 29.5-68.5). With regard to the beginning of the treatment, the median time since diagnosis was 40.0 days (range, 22.3-56.3). Of note, at the treatment initiation $51 \%(36 / 71)$ of the patients did not have their full driver mutations panel results available. 
Among the patients whose driver mutation profile was not available at the treatment initiation, $42 \%$ $(15 / 36)$ had a targetable alteration identified later on. The chemotherapy was replaced by a tyrosine kinase inhibitor (TKI) as soon as the molecular profile became available in $67 \%$ of the cases $(n=8 / 12)$. In two patients (17\%), the TKI was initiated after disease progression under chemotherapy regimen. Moreover, other 2 patients started TKI after disease progression and molecular profile characterization, simutaneously.

When assessing all 78 patients, a total of $29(37 \%)$ patients harbored a targetable alteration and just $46 \%(n=13 / 28)$ received a TKI as first line therapy. Furthermore, the median time to the TKI initiation was more than two times longer than any treatment initiation, 92.0 days (range, 45.0-234.0) versus 40.0 days (range, 22.3-56.3) (Figure 1E).

Finally, to evaluate in which step of driver mutations characterization a longer time was spent, we compared the median interval between the diagnosis and the testing request to the interval between the testing request and the testing results. We found, respectively, 29.0 versus 12.0 days $(p<0.001$, Wilcoxon Signed Ranks) (Figure 1F).

\section{Discussion}

In this study, we found EGFR mutation, ALK translocation, and ROS- 1 fusion in a proportion of $33 \%, 4 \%$, and $1 \%$, respectively. Even in a context of pharmaceutical industry-sponsored tests, many patients (61\%) did not have their molecular profile completely characterized. Moreover, at the treatment initiation, the full driver mutations panel results were not available in $51 \%$ of the patients. Considering those harboring targetable molecular alteration, $55 \%$ did not undego the targeted therapy upfront, as recommended.

Previous studies have investigated the access to biomarker testing and its rates over time in many countries, including Brazil ${ }^{8-10}$. However, to the best of our knowledge, the present study is the first to focus on the time spent in the molecular characterization and its impact on treatment choice in a realworld scenario of a middle income country.

According to The College of American Pathologists (CAP), it should take less than 14 days from the availability of a suitable sample to the report of its final results ${ }^{11}$. In our study, we showed a median of 12 days, which is under this recommendation. Besides, other retrospective studies showed that EGFR analysis lasts from 8 to 17 days in different countries ${ }^{12}$.

More recently, a Japanese publication demonstrated a median time of 11 days between the test ordering and its conclusion. The molecular test included EGFR, ALK, ROS1, and PD-L1. Among the patients harboring a targetable mutation, $93 \%$ underwent a directed therapy as the first-line ${ }^{13}$. These data contrasts with ours. In our analysis, only $52 \%$ of the patients had their full driver mutations panel results available at the time of the treatment initiation. It may be explained by logistical challenges that lead to the long interval between the diagnosis procedure and the testing request (median of 29 days). 
Awaiting biomarker testing results may delay treatment decisions in patients with advanced NSCLC, which may directly impact their clinical outcomes ${ }^{14}$.

This barrier to the personalized medicine implementation might be overcomed through the incorporating of reflex NSCLC biomarker testing at the level of the pathologist. Several studies had addressed the role of reflex testing in reducing the time between molecular investigation and treatment initiation. Phung et al. reported a reduction in this interval from 52 to around 23 days in a single-center study ${ }^{15}$. Similarly, a Canadian group demonstrated a shorter interval to the optimal first-line systemic therapy (median, 36 days [IQR, 16 to 91 days] versus 24 days [IQR, 8 to 43 days], $\mathrm{p}=.036$ ) with the reflex testing utilization ${ }^{16}$. Moreover, according to an institutional review, when EGFR/ALK results were available since the first consultation with the oncologist, nsNSCLC-patients had their time to treatment improved significantly (16 versus 29 days, $\mathrm{p}=.004)^{17}$.

Our study also revealed another concern regarding precision medicine incorporation among NSCLC patients in our setting. Although the multiple driver mutations already identified in nsNSCLC, the molecular panel was not complete in a majority of the patients. The six most important targetable mutations were investigated only in $39 \%$ of cases. Our data differs to the MYLUNG Cousortium, in which $49.0 \%$ of the patients were completely tested for these 5 biomarkers, with a tendency of improvement in this rate through the last years ${ }^{18}$.

Moreover, in our cohort, less than $4 \%$ of patients had their material evaluated through a NGS method, a technique recognized as fast and accurate. Thus, the use of NGS may also be time-sparing, which contributes to avoiding the initiation of the first-line treatment before the availability of all driver mutations testing results. Our study did not assessed ethnic differences in terms of access to NGS testing. A recent study showed that African Americans were less likely to undergo NGS testing when compared to those who are Caucasian $(39.8 \% \text { versus } 50.1 \%, \mathrm{p}<0.0001)^{19}$.

NGS testing may also improve clinical trial participation wich represents a very important pathway to access to innovative therapies. The same study previously cited showed that African American were also less likely to be treated in clinical trials $(1.9 \%$ versus $3.9 \%)$ due to the lack of access to NGS testing ${ }^{19}$.

Our study has some limitations. It was retrospective, and performed in a single-center, not reflecting the sociodemographic and genetic diversity of our population. Besides, we considered only the patients whose biomarker tests were requested. Thus, a selection bias could have occurred, since in daily practice many oncologists still use clinical predictors before requesting the tests. Maybe, it could justify the higher rate of EGFR mutations in the studied population (33.3\%), comparing to previous publications ${ }^{4}$.

Although the remarkable progress in NSCLC treatment until the mid-2010s, the landscape has been changing rapidly in the recent years. Thefore, the interval of patients recruitment would be considered too long to precisely assess the compliance to the targeted therapy, which is another limitation of this study. 


\section{Conclusions}

In patients with advanced nsNSCLC our data show a long median time between its diagnosis and the availability of the molecular profile report within medical practice, which may have influenced the choice of a non-personalized therapy as the first-line treatment. Indeed, in this study, the time between diagnosis and testing request was the longest step related to molecular characterization of these tumors. Together, these findings suggest that, even when testing reimbursement is not the main issue, other barriers in precision oncology implementation needed to be faced, such as availability of tumor samples and optimization of the processes involved in testing request, including the multidisciplinary care team training.

\section{Abbreviations}

NSCLC: non-small cell lung cancer

RWE: real world evidence

nsNSCLC: nonsquamous non small cell lung cancer

ANVISA: Brasilian Nacionial Health Suverillance Agency (Agência Nacional de Vigilância Sanitária)

NGS: next generation sequencing

TKI: tyrosine kinase inhibitor

CAP: College of American Pathologists

ECOG: Eastern Cooperative Oncology Group Scale of Performance Status

rtPCR: real time polymerase chain reaction

IHC: immunohistochemistry;

FISH: fluorescence in situ hybridization

\section{Declarations}

\section{Ethics approval and consent to participate}

This study was approved by an independent Ethics Committee (4.171.310), and the protocols were in accordance with the ethical guidelines of the 1975 Helsinki Declaration. Due to the retrospective nature of this study, the local Human Subjects Committee approved the waiver of participants' free and informed consent. 
Not applicable

Availability of data and materials

All data generated or analysed during this study are included in this published article.

Competing interestes

The authors declare that they have no competing interests.

\section{Funding}

The authors received no financial support in the design of the study, collection, analysis, and interpretation of data and in writing this manuscript.

\section{Authors' contributions}

The authors contributed equally to this work. All authors read and approved the final manuscript.

\section{Acknowledgements}

Not applicable

\section{References}

1. Bray F, Ferlay J, Soerjomataram I, Siegel RL, Torre LA, Jemal A. Global cancer statistics 2018: GLOBOCAN estimates of incidence and mortality worldwide for 36 cancers in 185 countries. CA Cancer J Clin. 2018 Nov;68(6):394-424. doi: 10.3322/caac.21492. Epub 2018 Sep 12. Erratum in: CA Cancer J Clin. 2020 Jul;70(4):313. PMID: 30207593.

2. Howlader N, Forjaz G, Mooradian MJ, Meza R, Kong CY, Cronin KA, Mariotto AB, Lowy DR, Feuer EJ. The Effect of Advances in Lung-Cancer Treatment on Population Mortality. N Engl J Med. 2020 Aug 13;383(7):640-649. doi: 10.1056/NEJMoa1916623. PMID: 32786189.

3. Ministério da Saúde. Instituto Nacional do Câncer José Alencar Gomes da Silva. Estimativa 2020 Incidência de cancer no Brasil [internet]. Rio de Janeiro; 2020. Available from:

https://www.inca.gov.br/sites/ufu.sti.inca.local/files/media/document/estimativa-2020-incidenciade-cancer-no-brasil.pdf. Acessed 22 Sep 2021.

4. Araujo LH, Baldotto C, Castro G Jr, Katz A, Ferreira CG, Mathias C, Mascarenhas E, Lopes GL, Carvalho H, Tabacof J, Martínez-Mesa J, Viana LS, Cruz MS, Zukin M, Marchi P, Terra RM, Ribeiro RA, Lima VCC, Werutsky G, Barrios CH; Grupo Brasileiro de Oncologia Torácica. Lung cancer in Brazil. J Bras Pneumol. 2018 Jan-Feb;44(1):55-64. doi: 10.1590/S1806-37562017000000135. PMID: $29538545 ;$ PMCID: PMC6104542.

5. Mok TS, Wu YL, Thongprasert S, Yang CH, Chu DT, Saijo N, Sunpaweravong P, Han B, Margono B, Ichinose Y, Nishiwaki Y, Ohe Y, Yang JJ, Chewaskulyong B, Jiang H, Duffield EL, Watkins CL, Armour 
AA, Fukuoka M. Gefitinib or carboplatin-paclitaxel in pulmonary adenocarcinoma. N Engl J Med. 2009 Sep 3;361(10):947 - 57. doi: 10.1056/NEJMoa0810699. Epub 2009 Aug 19. PMID: 19692680.

6. Rosell R, Carcereny E, Gervais R, Vergnenegre A, Massuti B, Felip E, Palmero R, Garcia-Gomez R, Pallares C, Sanchez JM, Porta R, Cobo M, Garrido P, Longo F, Moran T, Insa A, De Marinis F, Corre R, Bover I, Illiano A, Dansin E, de Castro J, Milella M, Reguart N, Altavilla G, Jimenez U, Provencio M, Moreno MA, Terrasa J, Muñoz-Langa J, Valdivia J, Isla D, Domine M, Molinier O, Mazieres J, Baize N, Garcia-Campelo R, Robinet G, Rodriguez-Abreu D, Lopez-Vivanco G, Gebbia V, Ferrera-Delgado L, Bombaron P, Bernabe R, Bearz A, Artal A, Cortesi E, Rolfo C, Sanchez-Ronco M, Drozdowskyj A, Queralt C, de Aguirre I, Ramirez JL, Sanchez JJ, Molina MA, Taron M, Paz-Ares L; Spanish Lung Cancer Group in collaboration with Groupe Français de Pneumo-Cancérologie and Associazione Italiana Oncologia Toracica. Erlotinib versus standard chemotherapy as first-line treatment for European patients with advanced EGFR mutation-positive non-small-cell lung cancer (EURTAC): a multicentre, open-label, randomised phase 3 trial. Lancet Oncol. 2012 Mar;13(3):239-46. doi: 10.1016/S1470-2045(11)70393-X. Epub 2012 Jan 26. PMID: 22285168.

7. Shaw AT, Kim DW, Nakagawa K, Seto T, Crinó L, Ahn MJ, De Pas T, Besse B, Solomon BJ, Blackhall F, Wu YL, Thomas M, O'Byrne KJ, Moro-Sibilot D, Camidge DR, Mok T, Hirsh V, Riely GJ, lyer S, Tassell V, Polli A, Wilner KD, Jänne PA. Crizotinib versus chemotherapy in advanced ALK-positive lung cancer. N Engl J Med. 2013 Jun 20;368(25):2385-94. doi: 10.1056/NEJMoa1214886. Epub 2013 Jun 1.

Erratum in: N Engl J Med. 2015 Oct 15;373(16):1582. PMID: 23724913.

8. Palacio S, Pontes L, Prado E, Arshad J, Ali R, Piha T, Bacchi CE, Mudad R, Lopes G. EGFR Mutation Testing: Changing Patterns of Molecular Testing in Brazil. Oncologist. 2019 Apr;24(4):e137-e141. doi: 10.1634/theoncologist.2018-0254. Epub 2018 Nov 16. PMID: 30446583; PMCID: PMC6459254.

9. Freitas HC, Torrezan GT, da Cunha IW, Macedo MP, Karen de Sá V, Corassa M, Ferreira ENE, Saito AO, Dal Molin GZ, Cordeiro de Lima VC, Carraro DM. Mutational Portrait of Lung Adenocarcinoma in Brazilian Patients: Past, Present, and Future of Molecular Profiling in the Clinic. Front Oncol. $2020 \mathrm{Jul}$ 2;10:1068. doi: 10.3389/fonc.2020.01068. PMID: 32714871; PMCID: PMC7343968.

10. Leal LF, de Paula FE, De Marchi P, de Souza Viana L, Pinto GDJ, Carlos CD, Berardinelli GN, Miziara JE, da Silva CM, Silva ECA, Pereira R, de Oliveira MA, Scapulatempo-Neto C, Reis RM. Mutational profile of Brazilian lung adenocarcinoma unveils association of EGFR mutations with high Asian ancestry and independent prognostic role of KRAS mutations. Sci Rep. 2019 Mar 1;9(1):3209. doi: 10.1038/s41598-019-39965-x. PMID: 30824880; PMCID: PMC6397232.

11. Lindeman NI, Cagle PT, Beasley MB, Chitale DA, Dacic S, Giaccone G, Jenkins RB, Kwiatkowski DJ, Saldivar JS, Squire J, Thunnissen E, Ladanyi M. Molecular testing guideline for selection of lung cancer patients for EGFR and ALK tyrosine kinase inhibitors: guideline from the College of American Pathologists, International Association for the Study of Lung Cancer, and Association for Molecular Pathology. J Thorac Oncol. 2013 Jul;8(7):823 - 59. doi: 10.1097/JTO.0b013e318290868f. Erratum in: J Thorac Oncol. 2013 Oct;8(10):1343. PMID: 23552377; PMCID: PMC4159960.

12. Lee $\mathrm{DH}$, Tsao MS, Kambartel KO, Isobe $\mathrm{H}$, Huang MS, Barrios $\mathrm{CH}$, Khattak $A$, de Marinis F, Kothari $\mathrm{S}$, Arunachalam A, Cao X, Burke T, Valladares A, de Castro J. Molecular testing and treatment patterns 
for patients with advanced non-small cell lung cancer: PIvOTAL observational study. PLoS One. 2018 Aug 27;13(8):e0202865. doi: 10.1371/journal.pone.0202865. PMID: 30148862; PMCID: PMC6110501.

13. Shimizu J, Masago K, Saito H, Nishino K, Kurata T, Itoh Y, Yoshimura Y, Yabuki Y, Dosaka-Akita H. Biomarker testing for personalized, first-line therapy in advanced nonsquamous non-small cell lung cancer patients in the real world setting in Japan: a retrospective, multicenter, observational study (the BRAVE study). Ther Adv Med Oncol. 2020 Feb 22;12:1758835920904522. doi: 10.1177/1758835920904522. PMID: 32127924; PMCID: PMC7036489.

14. Lim C, Tsao MS, Le LW, Shepherd FA, Feld R, Burkes RL, Liu G, Kamel-Reid S, Hwang D, Tanguay J, da Cunha Santos G, Leighl NB. Biomarker testing and time to treatment decision in patients with advanced nonsmall-cell lung cancer. Ann Oncol. 2015 Jul;26(7):1415-21. doi: 10.1093/annonc/mdv208. Epub 2015 Apr 28. PMID: 25922063.

15. Anand K, Phung TL, Bernicker EH, Cagle PT, Olsen RJ, Thomas JS. Clinical Utility of Reflex Ordered Testing for Molecular Biomarkers in Lung Adenocarcinoma. Clin Lung Cancer. 2020 Sep;21(5):437442. doi: 10.1016/j.cllc.2020.05.007. Epub 2020 May 13. PMID: 32600793.

16. Cheema PK, Menjak IB, Winterton-Perks Z, Raphael S, Cheng SY, Verma S, Muinuddin A, Freedman R, Toor N, Perera J, Anaka M, Victor JC. Impact of Reflex EGFR/ ALK Testing on Time to Treatment of Patients With Advanced Nonsquamous Non-Small-Cell Lung Cancer. J Oncol Pract. 2017 Feb;13(2):e130-e138. doi: 10.1200/JOP.2016.014019. Epub 2016 Dec 28. PMID: 28029301.

17. Lim C, Tsao MS, Le LW, Shepherd FA, Feld R, Burkes RL, Liu G, Kamel-Reid S, Hwang D, Tanguay J, da Cunha Santos G, Leighl NB. Biomarker testing and time to treatment decision in patients with advanced nonsmall-cell lung cancer. Ann Oncol. 2015 Jul;26(7):1415-21. doi: 10.1093/annonc/mdv208. Epub 2015 Apr 28. PMID: 25922063.

18. Robert NJ, Nwokeji E, Espirito JL, Chen L, Karhade M, Evangelist M, Spira A, Neubauer M, Bullock S, Coleman R. Biomarker Tissue Journey Among Patients with Untreated Metastatic Non-Small Cell Lung Cancer in the US Oncology Network Community Practices. J Clin Oncol. 2021 May; 39(15):9004-9004. doi:10.1200/JC0.2021.39.15_suppl.9004.

19. Bruno DS, Hess LM, Li XI, Su EW, Zhu TE, Patel M. Racial disparities in biomarler testing and clinical tral enrollment in non-small cell lung cancer (NSCLC). J Clin Oncol. 2021 May; 39 (15): 9005-9005. doi: 10.1200/JCO.2021.39.15_suppl.9005.

\section{Figures}




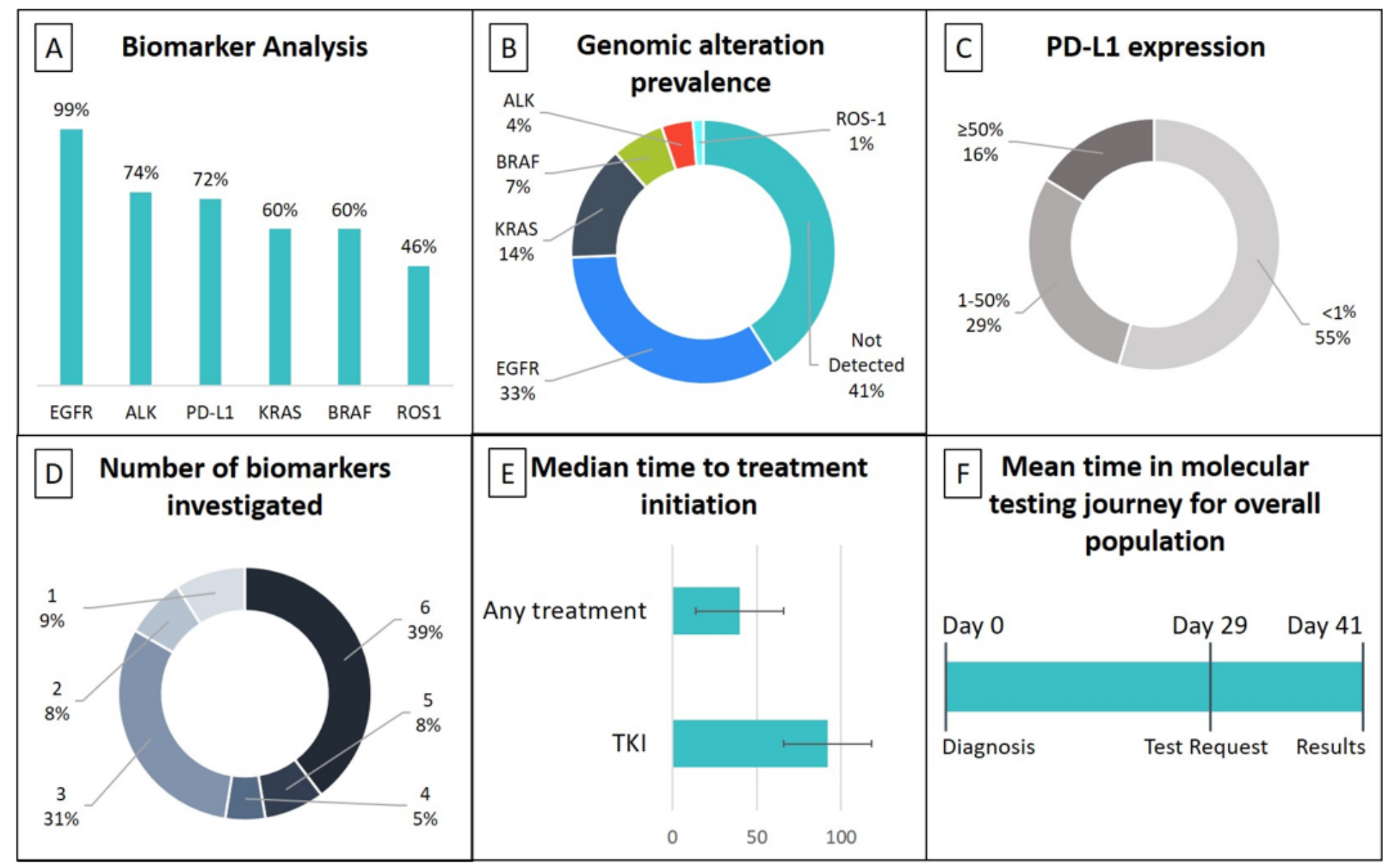

Figure 1

Biormaker analysis of NSCLC, genomic alteration prevalence and time in molecular testing journey of population. (1A) Frequency of testing according to the biomarker analyzed, (1B) genomic alteration prevalence in the study population, (1C) frequency of different categories of PDL-1 expression (i.e, PDL-1 $<1 \%, 1-49 \%, \geq 50 \%)$, (1D) number of biomarker tested in the same patient and its frequency, (1E) mean time to TKI initiation after EFGR characterization (in days) and mean time for any treatment initiation, (1F) mean time spent in the molecular testing journey since the advanced lung cancer diagnosis (in days). 